\title{
Frameless
}

Volume 1 | Issue 1

Article 18

November 2019

\section{A Comparison of Colorimetric Performance of Oculus and HTC Virtual Reality Headsets}

\author{
Morteza Maali Amiri \\ Rochester Institute of Technology, mm2391@rit.edu \\ Kamran Binaee \\ Rochester Institute of Technology, kb4000@rit.edu \\ James Ferwerda \\ Rochester Institute of Technology, jafpci@rit.edu
}

Follow this and additional works at: https://scholarworks.rit.edu/frameless

\section{Recommended Citation}

Amiri, Morteza Maali; Binaee, Kamran; and Ferwerda, James (2019) "A Comparison of Colorimetric Performance of Oculus and HTC Virtual Reality Headsets," Frameless: Vol. 1: Iss. 1, Article 18. DOI: 10.14448/Frameless.01.005

Available at: https://scholarworks.rit.edu/frameless/vol1/iss1/18

This Article is brought to you for free and open access by RIT Scholar Works. It has been accepted for inclusion in Frameless by an authorized editor of RIT Scholar Works. For more information, please contact ritscholarworks@rit.edu. 


\title{
FRAMEEESS
}

\section{A Comparison of Colorimetric Performance of Oculus and HTC Virtual Reality Headsets}

\author{
Morteza Maali Amiri* \\ Carlson Center for Imaging Science \\ Rochester Institute of Technology
}

\author{
Kamran Binaee \\ Carlson Center for Imaging Science \\ Rochester Institute of Technology
}

\author{
James Ferwerda \\ Carlson Center for Imaging Science \\ Rochester Institute of Technology
}

Abstract: The colorimetric characterization of the two virtual reality headsets, namely, HTC and Oculus are compared to each other. In order to do that, first, a colorimeter is used to measure the colorimetric values of the primary ramps in a darkened and controlled environment. It is observed that the two headsets behave more or less the same with HTC outputting an overall higher level of luminance and having more consistent right and left displays. Weighted regression is also used as a means to characterize the devices and the results are compared to the traditional method of colorimetric characterization showing the superiority of the weighted regression in this case. 
Vol. 1, No. 1 - 2019

Frameless

\section{INTRODUCTION}

The idea of virtual reality (VR) has been around for a long period of time, which goes back to even before computers were invented. The panoramic paintings from the $19^{\text {th }}$ century, can be regarded as the earliest effort of virtual reality. These paintings were intended to fill the viewer's entire vision field, making them present at some historical scene or event. Ivan Sutherland created the first VR head-mounted display system in 1965. Companies such as, Facebook, Sony, Google, and Samsung are probably the most famous ones in the VR industry [1]. Nowadays, VR systems have numerous different applications. The most important ones are training and education, marketing and design, research and game industry [2-5].

Basically, virtual reality is interactive experience that occurs inside a simulated environment generated by a computer. This environment incorporates mostly auditory and visual along with other types of sensory feedback [6]. The immersive environment could be the same as real world or a fictitious one. Virtual reality headsets are one of the most common technologies used nowadays to produce realistic sounds, images and other sensations simulating a physical presence of a user in an imaginary or virtual environment [6]. The colorimetric performance of these devices has been mostly neglected. However, if it is intended to use these devices for the purpose of psychophysical experiments they should be first colorimetrically characterized.

A substantial amount of research has been dedicated to the CRT and LCD colorimetry and assessing such properties as channel independence, spatial uniformity and independence [7-12]. The most important characteristics of the displays is optoelectronic transfer function, OETF. It is ordinarily a nonlinear function for computerbased devices [10]. CRTs, for instance, are characterized via models called gain-offset gamma. It should be noted that the same model cannot be used for LCDs. It is suggested that one-dimensional look-up tables (LUTs) be built for LCDs to change the digital counts to the radiometric scalars [10]. In the process of colorimetric characterization of the CRTs, flare should also be taken into accounts, which is either internal or external. The internal flares correspond to the display's black level [10].

In this work, the colorimetric performance of two virtual reality headsets are examined. The first one is Oculus headset and the second one is HTC. To be more thorough, the left and right displays in both devices are examined and the results in terms of the shape of the OETF function, the additivity for each channel, and the chromaticity of the primary ramps are reported. To the best of the authors' knowledge, this is the first work trying to colorimetrically characterize two virtual reality headsets in a comparative manner. Amiri and Fairchild described using the weighted regression to colorimetrically characterize a digital camera [13]. In this work, the same method is employed to characterize the virtual reality headsets and the results are compared to the traditional method proposed by Fairchild and Wyble [10]. 


\section{EXPERIMENT AND PROCEDURE}

Two VR headsets, i.e., Oculus DK2 and HTC VIVE, are used for this study.

To do the colorimetric measurements, the colorimeter is used. The colorimeter utilized is KONIKA-MINOLTA CS-100 A, which could measure CIEXYZ of the 2-degree standard observer.

To do the colorimetric characterization for the devices, 15 ramps of primaries are used and the output luminance along with the chromaticity are measured via the colorimeter. The environment in which the measurements are made is kept dark to make the light coming out of the headset the only existent one. The situation is indeed similar to when an observer is looking through the lens of the headsets at the displays. Therefore, the distance between the colorimeter and the lens of the headset is the same as that of the observer. In other words, the colorimeter is perpendicular to the center of the display. A function is then fitted to the measured luminance to figure out the OETF function of the displays. The additivity and also change in the chromaticities of the primary ramps are examined. The study is done in a comparative manner for the two headsets. The left and right displays along with the whole headsets are compared to each other.

At the end, two methods are used for colorimetric characterization of the headsets. The first method used is called Fairchild's method which is laid out in [10]. This method has been extensively used for the characterization purposes. The second method used is weighted regression. This method was proposed by [13], and it has been used for digital camera characterization.
It is worth going through the methods and explaining them here.

The first method, which is called Fairchild's method in this work, is briefly explained. First, three sets of Look-Up Tables (LUTs) are built for each R, G and B channels, which are generally built, using subsampled measurements and interpolation. These LUTs are used to change the digital counts to linear RGB (also called radiometric scalars). The LUTs are shows in Equation 1.

$$
\begin{aligned}
& \mathrm{R}=\operatorname{LUT}\left(\mathrm{d}_{\mathrm{r}}\right) \\
& \mathrm{G}=\operatorname{LUT}\left(\mathrm{d}_{\mathrm{g}}\right) \\
& \mathrm{B}=\operatorname{LUT}\left(\mathrm{d}_{\mathrm{b}}\right)
\end{aligned}
$$

where, $\mathrm{R}, \mathrm{G}$ and $\mathrm{B}$ are the scalars between 0 and 1 .

These scalars are then used in equation 2, to estimate the CIEXYZ of the samples.

$$
\begin{array}{ccc}
X & R & X \\
\lceil Y\rceil & =\mathbf{M}[G\rceil & +\lceil Y\rceil \\
Z_{\text {est }} & B_{\text {linear }} & Z_{\text {black }}
\end{array}
$$

$\mathbf{M}$ is the matrix of primaries shown in equation (3).

$\mathbf{M}=\left[\begin{array}{rrl}X_{r, \max } & X_{g, \max } & X_{b, \max } \\ Y_{r, \max } & Y_{g, \max } & Y_{b, \max }\end{array}\right]$

The matrix of primaries is formed using the CIEXYZ of the full-on R, G and B channels separately. The XYZ of the black is measured using the measurement made when the digital counts are set to zero. 
Weighted regression is in essence nonlinear regression that has a weighting factor considering how far or close testing sample is, compared to the training one.

In order to do the nonlinear regression, first RGB digital counts should be multiplied by each other as follows.

RGBtr $=[1 R G$
$B R G R B G B R^{2}$
$G^{2} B^{2} R G B R^{2} G$
$G^{2} B B^{2} R R^{2} B G^{2} R$
$\left.B^{2} G\right]^{T}$

After multiplying the RGB terms together, they are used in the following equation.

\section{M= XYZtr $\times \operatorname{pinv}($ RGBtr $)$}

where, RGBtr is the matrix that also contains the multiplication terms of RGB for learning samples, and $\mathbf{M}$ is the matrix containing the relationship between the CIEXYZ (shown by XYZtr) and RGB information of training samples. Therefore, by multiplying the matrix $\mathbf{M}$ by the matrix containing the RGB digital counts of testing samples (shown by RGBte) that also contains their multiplication the same as RGBtr, it is possible to obtain their CIEXYZ (denoted by XYZte in equation 6), shown in equation 6 . The nonlinear regression used is a polynomial with 17 coefficients, which leads to an acceptable result.

\section{XYZte $=$ M $\times$ RGBte}

where, XYZte is the CIEXYZ of the testing samples. It should be noted that the CIEXYZ of the training samples used in equation 5 is black subtracted. In the case of the weighted regression, first, the distance between the training and testing samples are calculated as shown in equation 7 .
$\Delta E_{E u}=\sqrt{\left(R_{-} T-R_{-} L\right)^{2}+\left(G_{-} T-G_{-} L\right)^{2}+\left(B_{-} T-B_{-} L\right)^{2}}$

where, $\Delta E_{E u}$ is the Euclidean distance, R_T, G_T and B T are the RGB digital counts of the testing samples and $R_{-} L, G_{-} L$ and $B \_L$ are the RGB digital counts of the learning samples.

The nonlinear regression is then enhanced herein through incorporating a weighting function into it. The weights are first calculated using equation (8).

$$
W=\frac{1}{\imath \Delta E i+s}
$$

where, $\Delta E i$ is the Euclidean distance between the RGB digital counts of the learning from testing samples as shown in equation 7. In order to also prevent the weights from becoming infinity in case $\Delta E$ is zero, $\mathrm{s}$ which is set to 0.01 is used. The distance is calculated between each testing sample and the whole learning set. Therefore, there are $\mathrm{n}$ different weights for each testing sample ( $\mathrm{n}$ is the number of training samples); now that the weights are computed, they are placed in a diagonal matrix as shown in equation 9 for each testing sample.

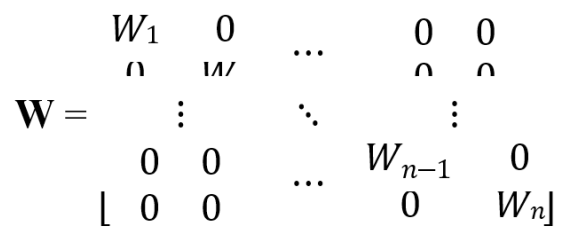

After arranging the weights for each testing sample in diagonal matrix as shown in equation (9), the weights are used in equation (10) as follows to calculate the matrix $\mathbf{M}$ between the CIEXYZ and RGB digital counts of learning samples.

$\mathbf{M}=\mathbf{X Y Z t r} \times \mathbf{W} \times \operatorname{pin} v(\operatorname{RGBtr} \times \mathbf{W})$

Therefore, matrix $\mathbf{M}$ would be specific to that particular testing sample. Matrix $\mathbf{M}$ is then 
multiplied by the testing samples' RGB digital counts resulting in their CIEXYZ. It should be noted that using any kind of weighted regression would lead to each testing sample having their own specific matrix $\mathbf{M}$.

Therefore, the difference between the Fairchild's and the nonlinear weighted regression is, the former needs the device to be colorimetrically well-defined, but the latter relies on math and does not care how good colorimetrically a device functions. In the weighted regression, all the experimenter has to do is to measure quite a few samples covering the color space of the device but in Fairchild's method, first, LUTs must be built and then the linearized digital counts are multiplied by the matrix of primaries. The matrix of primaries consists of measurements of red, green and blue primaries and an extreme level of caution should be taken to measure them. However, in the case of the weighted regression, a large number of samples are usually measured making the experimenter's level of thoroughness when it comes to measuring of less importance. In other words, the Fairchild's method put a lot of pressure on the experimenter to do the measurement as well as s/he could but in the case of weighted regression the same thing does not hold. These two methods are compared at the end by a final table of results. CIEDE2000 is used to do the comparison.

\section{RESULTS AND DISCUSSIONS}

Fig.1. shows the overall result of the characterization of the four displays.

As it is observed from Fig.1., the left and right displays in HTC match each other but it is not the case for the Oculus. The fact that the two displays behave the same in HTC and not in the Oculus have a significant impact on the observers' color perception. Green channels in both headsets has the highest luminance which makes sense, as the observers' highest sensitivity is to this color, and the device should be able to provide enough high energy in those areas of spectrum [12]. Blue, on the other hand, has the lowest intensity, which does not really matter due to the fact that humans' sensitivity in those area of spectrum is not significant [12]. Overall, the higher luminance of the HTC could make an observer have a better experience in terms of the contrast perception and colorfulness of the objects virtually shown on the displays [12].

In this section, the additivity of the displays is examined. The additivity is tested through measuring the white point directly and comparing it to the CIEXYZ of the white point calculated through adding up the XYZ of the $\mathrm{R}, \mathrm{G}$ and $\mathrm{B}$ channels. Table 1 shows the CIEXYZ of the white measured directly and the CIEXYZ of the added R, G and B channels. It should be noted that the additivity should be checked after the black subtraction.

As observed from Table 1, it is obvious that Oculus headset is better in terms of following the additivity rule. Both headsets show inconsistency between their right and left displays.

The chromaticities of the color ramps used in this work are now examined, once before the black subtraction and once after that for all the displays. Fig.2. shows the chromaticities of the primary ramps.

As it is observed from Fig.2, there is still variation around all the primaries even after the black subtraction. Ideally, the ramps 
Vol. 1, No. 1 - 2019

Frameless
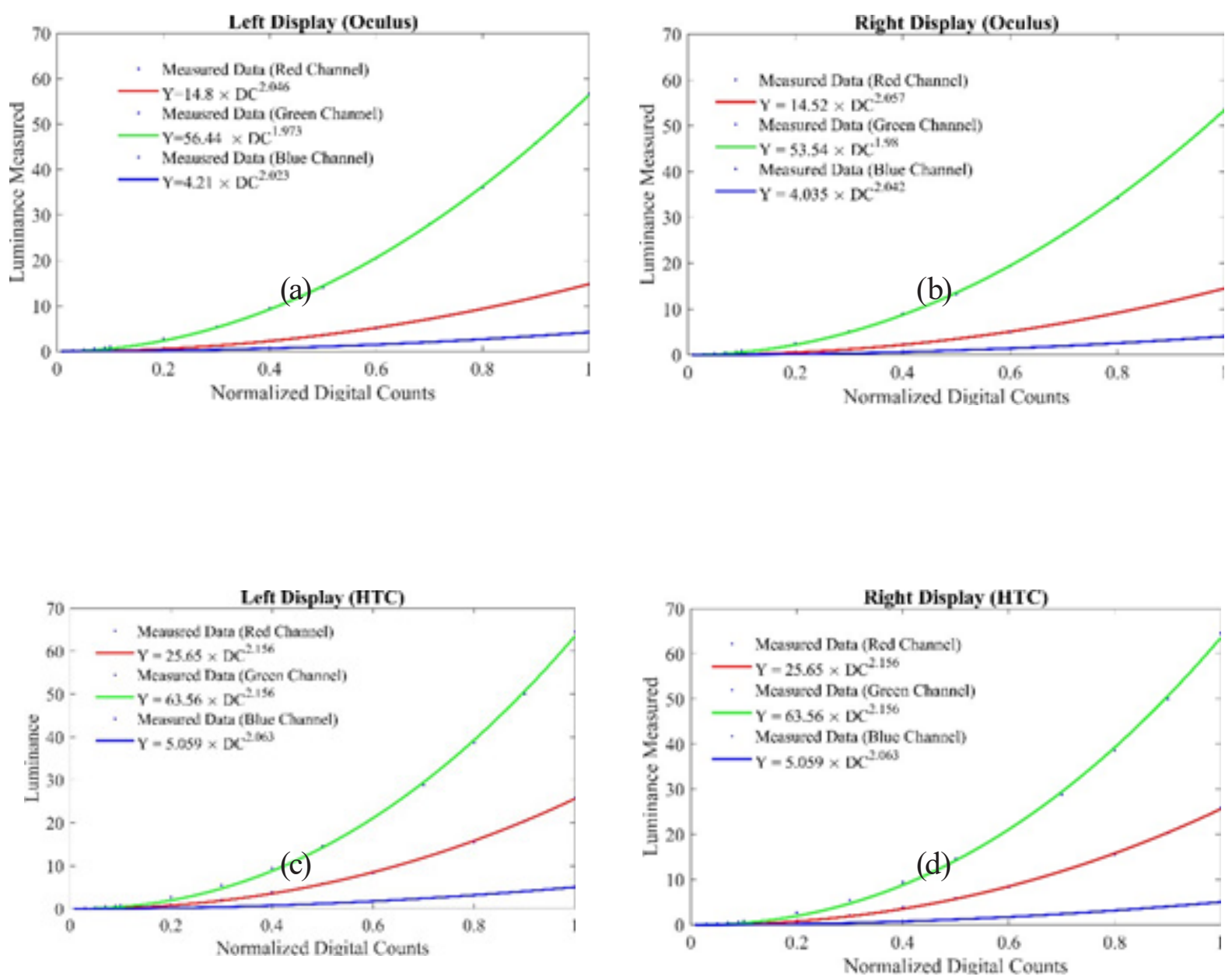

Fig.1. Results of the colorimetric characterizations. 
Table 1. CIEXYZ of the white measured directly and the sum of $R, G$ and $B$ channels.

\begin{tabular}{ccccccc}
\hline Display & $\mathrm{X}_{\text {white }}$ & $\mathrm{Y}_{\text {white }}$ & $\mathrm{Z}_{\text {white }}$ & $\mathrm{X}_{\text {sum }}$ & $\mathrm{Y}_{\text {sum }}$ & $Z_{\text {sum }}$ \\
\hline Left Oculus & 61.6084 & 71.1300 & 77.2989 & 64.4967 & 75.6900 & 81.2793 \\
Right Oculus & 59.9274 & 68.9300 & 74.8072 & 62.3692 & 72.3200 & 78.5001 \\
Left HTC & 76.5249 & 86.4200 & 99.9536 & 82.0886 & 95.5400 & 102.5065 \\
Right HTC & 90.6872 & 102.5600 & 115.0711 & 94.5760 & 110.6100 & 115.7326 \\
\hline
\end{tabular}

should be formed into a point on the chromaticity diagram, however, herein it is not the case. It might show that the black subtraction is not the only source of noise that the experimenter should be worried about in the case of these headsets. In CRT displays, a simple back subtraction always works but herein it is not as simple.

It is worthwhile to also implement weighted regression along with the classical method of colorimetric characterization (referred to as Fairchild's method) to see how accurate the colorimetric color reproduction in the case of these headsets is. Table 2 shows the results using these two methods.

As it is observed, Fairchild's method has failed to perform as well as weighted regression. This makes sense considering that the headsets are not colorimetrically well specified as shown in the prior table and figures; therefore, Fairchild's method, which assumes that the device is colorimetircally well determined would fail in these cases. On the other hand, weighted regression would not care about the colorimetric performance of the device and only put a weight on the samples based on how far they are from a sample in training set. It should be noted that weighted regression takes longer amount of time to run than the Fairchild's approach.

Overall, it is obvious that the two headsets are not colorimetrically well defined as compared to the CRTs or LCDs and when they are used for the purpose of psychophysical experiments, a greater deal of caution should be exerted by the experimenter.

Table 2. Colorimetric color reproduction accuracy using different approaches.

\begin{tabular}{ccccccc}
\hline \multirow{2}{*}{ Display } & \multicolumn{3}{c}{ Fairchild's Method } & \multicolumn{3}{c}{ Weighted Regression } \\
\cline { 2 - 7 } & Mean & Max & Med & Mean & Max & Med \\
\hline Left Oculus & 1.6428 & 2.5032 & 1.9487 & 1.1318 & 2.0452 & 0.6631 \\
Right Oculus & 3.2192 & 4.2557 & 3.5202 & 2.6688 & 3.1432 & 0.9272 \\
Left HTC & 3.4098 & 6.0158 & 2.2300 & 1.7008 & 4.5321 & 1.0170 \\
Right HTC & 3.8917 & 6.2341 & 3.2265 & 1.9030 & 4.9029 & 0.9178 \\
\hline
\end{tabular}


Vol. 1, No. 1 - 2019

Frameless

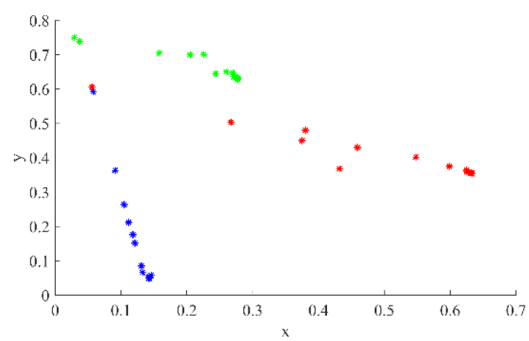

(a)

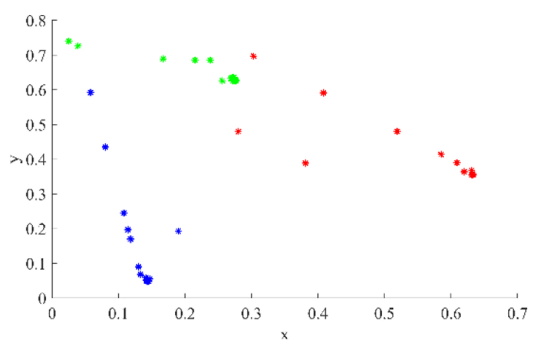

(c)

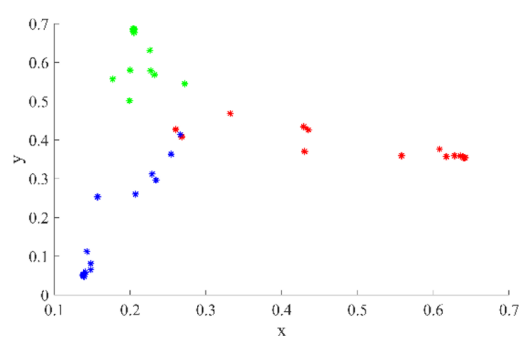

(e)

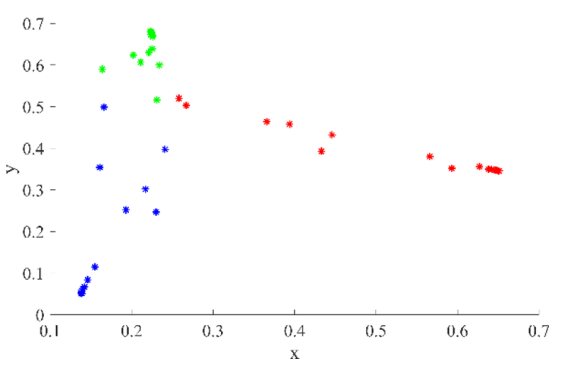

(g)

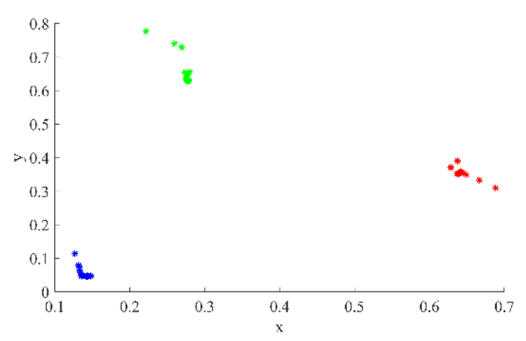

(b)

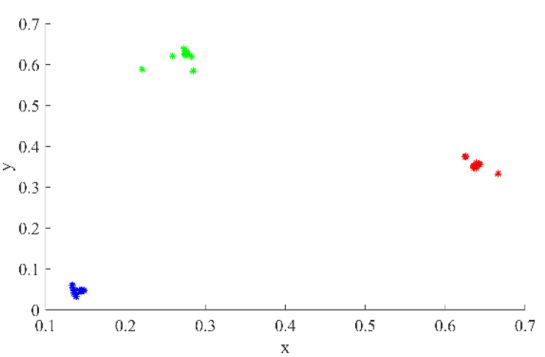

(d)

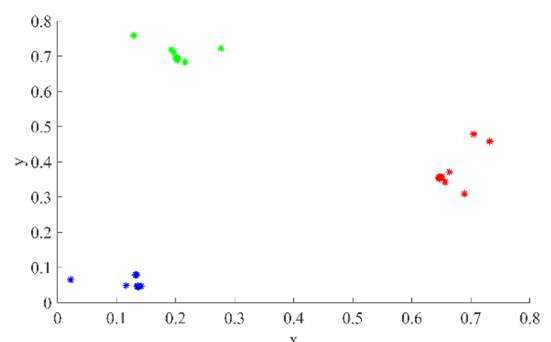

(f)

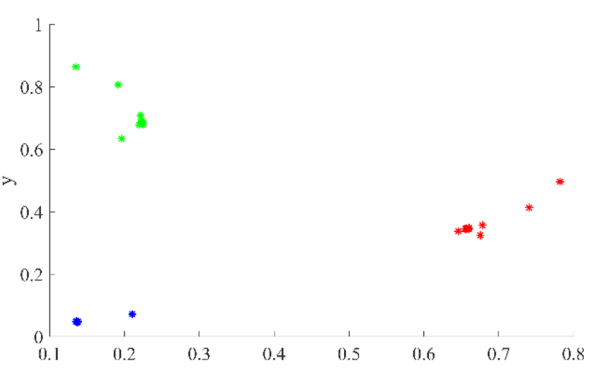

(h)

Fig.2. Chromaticities of (a) Oculus left display before black subtraction (b) Oculus left display after black subtraction (c) Oculus right display before black subtraction (d) Oculus right display after black subtraction (e) HTC left display before black subtraction (f) HTC left display after black subtraction (g) HTC right display before black subtraction (h) HTC right display after black subtraction. 


\section{CONCLUSIONS}

A comparison was drawn between the HTC and Oculus virtual reality headsets. Quite a few ramps of primaries were measured via a colorimeter. It was ascertained that the two headsets act almost the same with HTC having a higher level of luminance output and more consistent right and left displays. Two different methods were also used for colorimetric characterization of the devices. It was demonstrated that weighted regression led to a better result compared to a traditional method usually used for LCDs.

\section{REFERENCES}

1. C, Judd. "A Brief History of Virtual Reality." Lowdown.carphonewarehouse. com, 22 Oct. 2015, lowdown. carphonewarehouse.com/news/a-briefhistory-of-virtual-reality/29235/.

2. Burdea Grigore, C., and P. Coiffet. Virtual reality technology . London: WileyInterscience, 1994.

3. Algharabat, Raed, Amjad Abu El-Samen, and Charles Dennis. "The Effects of 3D Telepresence and 3D authenticity on the Online Retailer." Advances in Marketing (2011): 21.

4. Binaee, Kamran, and Gabriel J. Diaz. "Assessment of an augmented reality apparatus for the study of visually guided walking and obstacle crossing." Behavior research methods (2018): 1-9.
5. https://www.newsweek.com/virtual-realityfuture-film-461829

6. https://en.wikipedia.org/wiki/Virtual_reality

7. Berns RS, Katoh N. Methods for characterizing displays. in Green $P$ and MacDonald LW, editors, Color Engineering: Achieving Device Independent Colour, England: John Wiley \& Sons; 2002. p 127-164.

8. Katoh N, Deguchi T, Berns RS. An accurate characterization of CRT monitor (II) proposal for an extension to CIE method and its verification. Opt Rev 2001;8:397-408.

9. Katoh N, Deguchi T, Berns RS. An accurate characterization of CRT monitor (I) verification of past studies and clarifications of gamma. Opt Rev 2001;8:305-314.

10. Fairchild MD,Wyble DR. Colorimetric characterization of the Apple Studio Display (Flat Panel LCD). Munsell Color Science Laboratory Technical Report 1998, http:// www.cis.rit.edu/mcsl/research/PDFs/LCD.pdf

11. E. A. Day, L. Taplin, R. S. Berns, Colorimetric Characterization of a Computer-Controlled Liquid Crystal Display, 2004;29:365-373.

12. M. D. Fairchild, Color Appearance Models, 3rd edition, Wiley, 2013.

13. M. M. Amiri, M. D. Fairchild, A strategy toward spectral and colorimetric color reproduction using ordinary digital cameras, Color Research and Application, 43, 675-684 (2018). 